\title{
Is Embolization an Effective Treatment for Recurrent Hemorrhage After Hip or Knee Arthroplasty?
}

\author{
Peter I. Kalmar MD $@$, Andreas Leithner MD, Reinhard Ehall MD, \\ Rupert Horst Portugaller MD
}

Received: 1 May 2015/Accepted: 22 July 2015/Published online: 29 July 2015

(C) The Association of Bone and Joint Surgeons (B) 2015

\begin{abstract}
Background Spontaneous recurrent hemorrhage after arthroplasty of the hip or knee is a rare condition. In patients who do not have coagulopathy, the likeliest etiology for hemarthrosis is hypertrophic vascular synovium. Treatments include arthroscopic or open synovectomy, or angiography with embolization; however, because the condition is rare, seldom reported, and debilitating, small case series characterizing the efficacy of any approach are
\end{abstract}

Each author certifies that he or she, or a member of his or her immediate family, has no funding or commercial associations (eg, consultancies, stock ownership, equity interest, patent/licensing arrangements, etc) that might pose a conflict of interest in connection with the submitted article.

All ICMJE Conflict of Interest Forms for authors and Clinical Orthopaedics and Related Research ${ }^{\circledR}$ editors and board members are on file with the publication and can be viewed on request.

Clinical Orthopaedics and Related Research ${ }^{\circledR}$ neither advocates nor endorses the use of any treatment, drug, or device. Readers are encouraged to always seek additional information, including FDAapproval status, of any drug or device prior to clinical use.

Each author certifies that his or her institution approved the human protocol for this investigation, that all investigations were conducted in conformity with ethical principles of research, and that informed consent for participation in the study was obtained.

P. I. Kalmar ( $)$, R. H. Portugaller

Department of Radiology, Medical University of Graz,

Auenbruggerplatz 9, 8036 Graz, Austria

e-mail: peter.kalmar@medunigraz.at

\section{A. Leithner}

Department of Orthopedics, Medical University of Graz, Graz,

Austria

R. Ehall

Department of Orthopedics, State Hospital Bad Radkersburg,

Bad Radkersburg, Austria important to allow a collective experience with this condition to emerge.

Questions/purposes We attempted to (1) determine whether angiography with embolization can prevent recurrent hemarthrosis after hip or knee arthroplasty in a small series of patients with or without coagulopathy, and (2) evaluate complications associated with this intervention.

Methods Between 2005 and 2014, seven patients presented with spontaneous, recurrent hemarthroses. One patient had polycythemia vera and factor XIII deficiency as underlying illnesses. All patients were treated with selective transarterial embolization, and all had followup at a minimum of 12 months (range, 12-102 months; median, 74 months). Other treatments during this time included hematoma removals and flap operations in one patient, and indications for transarterial embolization included recurrent hemarthrosis. The patients included six men and one woman with a median age of 72 years (range, $61-78$ years). Five patients underwent one or more reoperations before transarterial embolization. A diagnostic workup for coagulopathy was performed in all patients; one patient was identified to have polycythemia ruba vera and factor XIII deficiency, however the patient still was treated with transarterial embolization because it was perceived to be the least invasive of available options. No other patients had a diagnosis of coagulopathy. Angiography showed hypervascularity in all patients and a contrast agent showed extravasation in two. Selective transarterial embolization of branches of the internal iliac artery, common femoral artery, deep femoral artery, or the popliteal artery was performed with polyvinyl alcohol particles, microspheres, and/or coils. Patients were followed clinically during 12 to 102 months (median, 74 months) to determine whether the hemorrhages recurred. 
Results Technical success was achieved in all patients. No procedure-related complications were reported. On followup, recurrent hemorrhage was reported in one patient who had a diagnosis of coagulopathy before the procedure. He underwent three reinterventions and five reoperations. Three months after initial embolization, a flap procedure was performed.

Conclusions In a small series of patients with a minimum followup of 1 year, we found selective transarterial embolization to be effective in patients without underlying coagulopathy in preventing recurrences of spontaneous recurrent hematoma or hemarthrosis of the hip and the knee. This condition is rare, therefore comparative trials are unlikely to be done. Because transarterial embolization is relatively low risk and generally well tolerated, we consider it to be a reasonable approach for consideration with other options such as arthroscopic or open synovectomy and revision arthroplasty.

Level of Evidence Level IV, therapeutic study.

\section{Introduction}

Spontaneous recurrent hemorrhage is a rarely reported complication after hip or knee arthroplasty [6, 10, 12]. Although the incidence is greater in patients with coagulopathy, bleeding from hypertrophic vascular synovium is assumed to be the likeliest mechanism [1,8]. Other causes are pseudoaneurysm formation, arteriovenous fistula, and pigmented villonodular synovitis of the knee $[4,8]$. The interval between implantation and hemorrhage often is short, leading to reoperations before discharge. There also are reports of long intervals up to 12 years between episodes of bleeding $[6,10]$. Nonsurgical treatments, such as observation, compression, and workup for coagulopathy generally are reasonable initial approaches for a first-time hemarthrosis; recurrent hemarthroses usually warrant intervention because of the pain and disability involved.

More-invasive options include angiography with embolization, arthroscopic or open synovectomy, and revision arthroplasty $[1,3,9]$. However, because the condition is rare, seldom reported, and debilitating, small case series characterizing the efficacy of any approach are important to allow a collective experience with this condition to emerge. Waldenberger et al. evaluated this diagnosis [11], but they did not focus exclusively on patients with joint replacement with followups of at least 12 months.

We therefore sought to determine whether angiography with embolization can prevent recurrent hemarthrosis after hip or knee arthroplasty in a small series of patients, and to evaluate complications associated with this intervention.

\section{Patients and Methods}

Institutional ethical committee approval was obtained.

\section{Participants and Study Subjects}

Between January 2005 and September 2014, seven patients presented with spontaneous, recurrent hemarthroses. A diagnostic workup for coagulopathy was performed in all patients; one patient was identified to have polycythemia rubra vera and factor XIII deficiency, but was treated with transarterial embolization because it was perceived to be the least invasive of available options. No other patients were diagnosed with coagulopathy. All patients were treated with selective transarterial embolization and all had followup at a minimum of 12 months (range, 12-102 months; median, 74 months). Other treatments during this time included hematoma removals and flap operations in one patient. Indications for transarterial embolization during this period included recurrent hemarthrosis. The patients included six men and one woman with a median age of 72 years (range, 61-78 years).

Three patients underwent hip arthroplasty and four underwent knee arthroplasty. The median interval between implantation and first reoperation or endovascular embolization was 23 months (range, 2-156 months) (Table 1).

The diagnosis of recurrent hemarthrosis is made easily after TKA using arthrocentesis. After hip arthroplasty, the diagnosis is not easy to make, and was made in different ways in each of the three patients who had hip arthroplasties in this series. In one patient, the diagnosis of late hemarthrosis was made after MRI that was done owing to local swelling. This diagnosis was confirmed during a revision procedure at an outside center. Four days after the last procedure the patient was transferred to our center for embolization owing to continuous bleeding; the diagnosis of infection was excluded based on histologic analysis. In the second patient, swelling for several months led to the clinical diagnosis of a dehiscence of the fascia lata resulting in an operation where a local hematoma was identified and drained; however, recurrent swelling occurred, leading to subsequent operative revision 14 days later, eventually resulting in the embolization at our center 5 days later. In the third patient with hip arthroplasty, CT of the pelvis was performed because of recurrent pain and swelling of the left leg for 15 months. This revealed a suspected hematoma in the iliac muscle with potential communication to the hip. A subsequent MRI showed hemarthrosis communicating with a pelvic hematoma.

Four patients underwent one or more reoperations at peripheral hospitals and then were transferred to our tertiary referral center for embolization. Arthrocentesis was 


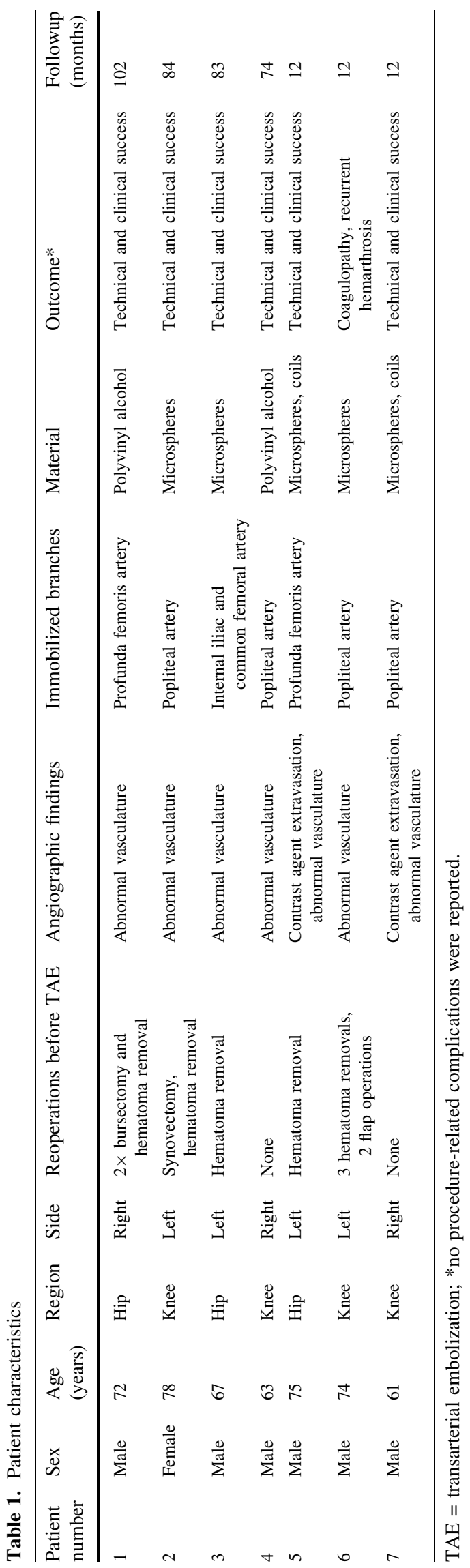

performed in all patients to document hemarthrosis. No signs of pigmented villonodular synovitis were found at primary (Patients 1-7) or revision surgery (Patients 1-3, 5, and 6). Routine workup for coagulopathy included blood cell count, prothrombin time, and international normalized ratio. One patient had elevated C-reactive protein levels $(15 \mathrm{mg} / \mathrm{L}) 1$ day before intervention. Median international normalized ratio before intervention was 0.96 (range, $0.90-1.19$ ), and the median hemoglobin level before embolization was $12 \mathrm{~g} / \mathrm{dL}$ (range, 9-13 g/dL).

Two embolizations were performed in the left hip, the right knee, and the left knee, respectively. One embolization was performed in the right hip. Preinterventional CT angiography was performed in two patients and MR angiography was performed in one.

Vascular access was performed with ipsilateral femoral arterial access in three patients and with contralateral access in five. Sedoanalgesia was used in three patients and local anesthesia was used in all patients. After placement of a 6-French catheter sheath (Cordis Corporation, Fremont, CA, USA), access to the contralateral common iliac artery was gained using 0.035 -inch hydrophilic guidewires and 4- to 5-French catheters (Terumo, Tokyo, Japan). To identify extravasation and/or areas of hypervascular tissue, initial nonselective angiography of the iliac, femoral, and popliteal arteries in two or more projections was performed using Ultravist ${ }^{\circledR} 300$ contrast agent ( $300 \mathrm{mg} \mathrm{I} / \mathrm{mL}$; Bayer, Berlin, Germany). Contrast agent volume was documented. Microcatheter selection of iliac, femoral, or geniculate arterial branches that extravasation, hypervascularity, or "tumor blush"-type vascularity was performed. Angiography showed hypertrophic branch arteries or abnormal vasculature in all patients and arterial extravasation in two (Fig. 1A). Median contrast agent use was $120 \mathrm{~mL}$ (range, 110-135 mL). No aneurysms, arteriovenous fistulae, tumors, or arteriovenous malformations were identified. Careful embolization was performed to the extent of nearstasis to the site of abnormal vasculature with polyvinyl alcohol particles ranging in size from $250 \mu \mathrm{m}$ to $500 \mu \mathrm{m}$ (Contour $^{\text {TM}}$; Boston Scientific, Natick, MA, USA) or microspheres ranging in size from $400 \mu \mathrm{m}$ to $700 \mu \mathrm{m}$ (Embozene ${ }^{\circledR}$; CeloNova Biosciences, San Antonio, TX, USA). Depending on the presence of extravasation, coils ranging from $2 \mathrm{~mm}$ to $3 \mathrm{~mm}$ (Cook Medical, Bloomington, IN, USA; or Boston Scientific) also were implanted to occlude the vessel proximal and distal to the bleeding site. Embolization was performed in branches of the internal iliac artery and the common femoral artery in one patient, the profunda femoris artery in two, and the popliteal artery in four. The median number of arteries treated per patient was three (range, 2-5 arteries). Microspheres were used in three patients, polyvinyl alcohol particles in two, and coils combined with microspheres in two. 

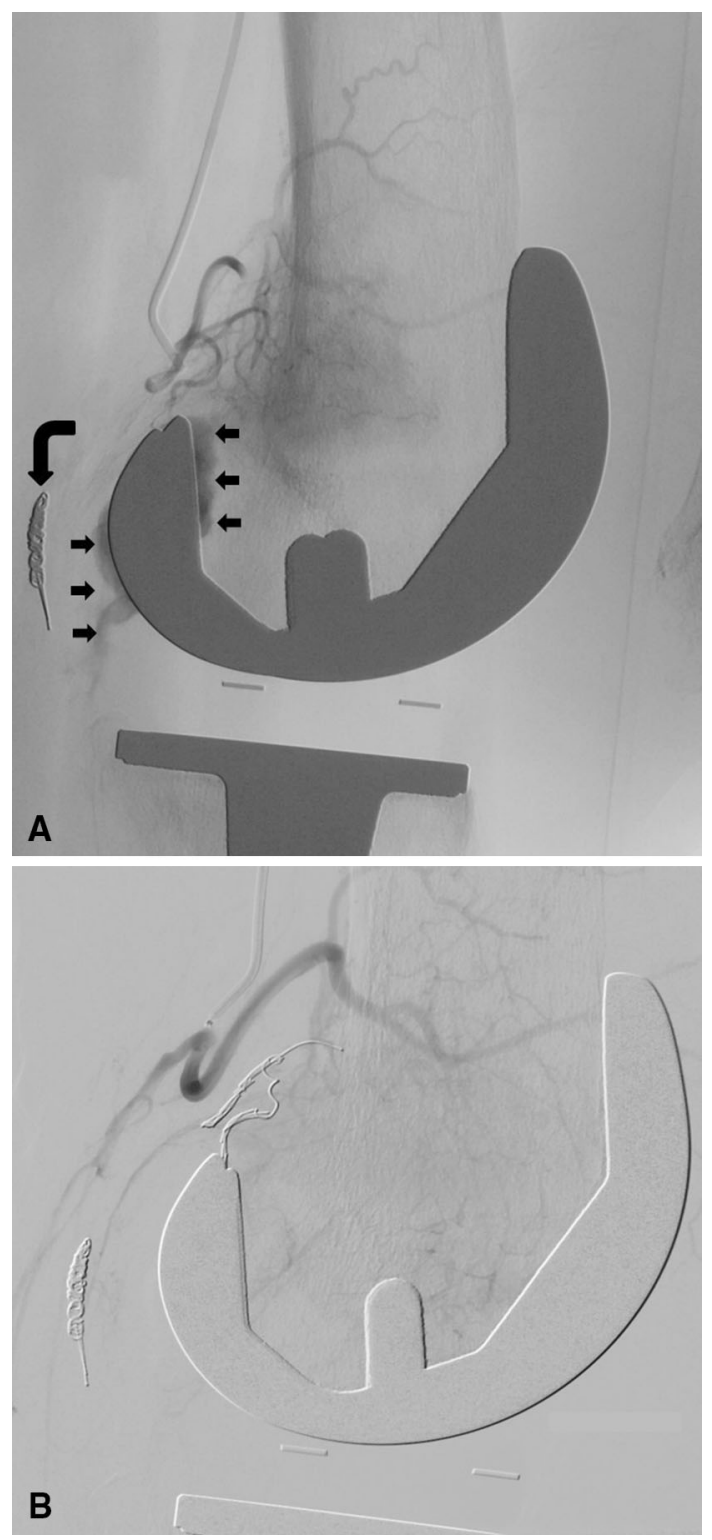

Fig. 1A-B A 74-year-old man had recurrent hemorrhage after total knee replacement. The patient had a diagnosis of polycythemia vera and factor XIII deficiency. (A) Owing to ongoing hemarthrosis, digital subtraction angiography was performed 6 days after initial coil embolization (curved arrow). Popliteal angiography showed a large extravasation in the popliteal fossa (arrows). (B) Complete cessation was seen after embolization.

In angiography, abnormal and increased vasculature frequently identifies the bleeding site.

\section{Aftercare}

Patients were followed clinically to evaluate for resolution of the hemarthroses and for recurrences. No patients were lost to followup.

\section{Results}

No procedure-related complications were reported. In six patients no recurrent hemarthrosis was observed at a median followup of 74 months (range, 12-102 months). One patient experienced severe recurrent hemarthrosis after 5 days. He subsequently underwent three endovascular reinterventions and five reoperations, including wound débridements and repeat closures. This patient was a 74-year-old man with polycythemia vera and factor XIII deficiency; he had undergone a reoperation before embolization. Three months after initial embolization, a flap procedure was performed with favorable healing response.

The patient who had elevated C-reactive protein levels 1 day before intervention had an infection and subsequent empyema develop at the treated joint. Two negative cultures of preinterventional aspirates were obtained 5 days and 3 days before transarterial embolization. The patient underwent empyema drainage 2 weeks after embolization. Owing to recurrent infections, his prosthesis was revised 4 months later.

\section{Discussion}

Spontaneous hemarthrosis after hip or knee arthroplasty is rare, but it can be painful and debilitating [2, 3, 10, 11]. The timing is variable, occurring from days to years after the index arthroplasty, as we observed and as prior studies have suggested [6, 10]. Initial management usually is nonoperative, but for patients whose hemarthrosis recurs, and who do not have a treatable coagulopathy, more-invasive options include arthroscopic or open synovectomy, revision arthroplasty, and transarterial embolization $[1,3$, 9]. Waldenberger et al. [11] evaluated this method, but they did not focus on patients with joint replacements with longer followups. In our small series, we found transarterial embolization to be generally well-tolerated and effective in most patients in preventing additional recurrences of intraarticular bleeding.

The main limitation of our study was the small number of patients, which is attributable to the rarity of this condition. Comparative studies are unlikely to be done because the condition is so uncommon, and case series therefore add to our collective understanding of the options for treatment. Other limitations include the relatively short followup (minimum of 1 year), which leaves open the possibility that these hemarthroses can recur, and the heterogeneity of the patient population in terms of diagnoses and presentations, which are typical of the condition, in our experience. 
During the last decade, case reports and other series reported satisfying results after transarterial embolization $[1,3,5,7,9]$. We found that transarterial embolization was well-tolerated and effective in six of seven patients; this tends to confirm and extend that experience. To our knowledge, our median followup was longer than those of the other studies. The repeated failure of endovascular and surgical therapy in a patient who had a known complex and rare coagulopathy showed the difficulties that this condition can cause. This concurs with a study by Sikkema et al. [10] of patients with hemophilia. They reported no difference in implant survival compared with survival in control subjects without hemophilia, although more hemarthroses were seen. The underlying illness was diagnosed after primary joint replacement and the thrombocytopenia was attributable to splenomegaly. The decision to perform transarterial embolization was decided interdisciplinarily owing to the lower invasiveness of this method. Our study tended to agree with that of Waldenberger et al. [11]; we found similar technical and clinical success, and together with that earlier study, we believe this approach can be considered alongside other less-invasive approaches available for treating patients with this complication.

When selecting embolizing agents we usually recommend the use of polyvinyl alcohol particles or microspheres, depending on the interventionalist's experience and preference. If extravasation is detected, coils should be used to occlude the vessel proximal and distal to the bleeding site.

None of the patients had procedure-related complications, such as ischemia or skin or tissue necrosis, which compares well with the findings of Waldenberger et al. [11]. Bagla et al. [1], in a study on geniculate artery embolizations in five patients, reported two cases of transient cutaneous ischemia. Compared with the literature, our study showed a similar technical and clinical success rate of $100 \%$ in all patients without known coagulopathy. Our study can be seen as a confidence builder by adding to the literature longer followup times in a rare patient collective. In a small series of patients with a minimum followup of 1 year, we found selective transarterial embolization to be effective in patients without underlying coagulopathy at preventing recurrences of spontaneous recurrent hematoma or hemarthrosis of the hip and the knee. This condition is rare, therefore comparative trials are unlikely to occur.
Because this procedure is relatively low risk and generally well tolerated, we consider transarterial embolization a reasonable approach to be considered alongside other options such as arthroscopic or open synovectomy and revision arthroplasty.

Acknowledgments We thank Hannes A. Deutschmann MD, of the Department of Radiology, Medical University of Graz, for contributing to this study.

\section{References}

1. Bagla S, Rholl KS, van Breda A, Sterling KM, van Breda A. Geniculate artery embolization in the management of spontaneous recurrent hemarthrosis of the knee: case series. $J$ Vasc Interv Radiol. 2013;24:439-442.

2. Dhondt E, Vanhoenacker FM, D'Archambeau O, Snoeckx A, Defreyne L. Angiographic findings and therapeutic embolization of late hemarthrosis after total joint arthroplasty. Skeletal Radiol. 2009;38:31-36.

3. Given MF, Smith P, Lyon SM, Robertson D, Thomson KR. Embolization of spontaneous hemarthrosis post total knee replacement. Cardiovasc Intervent Radiol. 2008;31:986-988.

4. Insall J, Scott WN, Ranawat CS. The total condylar knee prosthesis: a report of two hundred and twenty cases. J Bone Joint Surg Am. 1979;61:173-180.

5. Katsimihas M, Robinson D, Thornton M, Langkamer VG. Therapeutic embolization of the genicular arteries for recurrent hemarthrosis after total knee arthroplasty. J Arthroplasty. 2001;16:935-937.

6. Kindsfater K, Scott R. Recurrent hemarthrosis after total knee arthroplasty. J Arthroplasty. 1995;10(suppl):S52-55.

7. Maheshwari R, Kelley SP, Langkamer VG, Loveday E. Spontaneous recurrent haemarthrosis following unicompartmental knee arthroplasty and its successful treatment by coil embolisation. Knee. 2004;11:413-415.

8. Oishi CS, Elliott ML, Colwell CW Jr. Recurrent hemarthrosis following a total knee arthroplasty. J Arthroplasty. 1995;10 (suppl):S56-58.

9. Pritsch T, Pritsch M, Halperin N. Therapeutic embolization for late hemarthrosis after total knee arthroplasty: a case report. J Bone Joint Surg Am. 2003;85:1802-1804.

10. Sikkema T, Boerboom L, Meijer K. A comparison between the complications and long-term outcome of hip and knee replacement therapy in patients with and without haemophilia; a controlled retrospective cohort study. Haemophilia. 2011;17: 300-303.

11. Waldenberger P, Chemelli A, Hennerbichler A, Wick M, Freund MC, Jaschke W, Thaler M, Chemelli-Steingruber IE. Transarterial embolization for the management of hemarthrosis of the knee. Eur J Radiol. 2012;81:2737-2740.

12. Worland RL, Jessup DE. Recurrent hemarthrosis after total knee arthroplasty. J Arthroplasty. 1996;11:977-978. 